\title{
Keldysh action of a multiterminal time-dependent scatterer
}

\author{
I. Snyman \\ Institut-Lorentz, Universiteit Leiden, P.O. Box 9506, 2300 RA Leiden, The Netherlands
}

\section{Y. V. Nazarov}

Kavli Institute of Nanoscience, Delft University of Technology, 2628 CJ Delft, The Netherlands

(Received 15 January 2008; revised manuscript received 19 March 2008; published 14 April 2008)

\begin{abstract}
We present a derivation of the Keldysh action of a general multichannel time-dependent scatterer in the context of the Landauer-Büttiker approach. The action is a convenient building block in the theory of quantum transport. This action is shown to take a compact form that only involves the scattering matrix and reservoir Green's functions. We derive two special cases of the general result, one valid when reservoirs are characterized by well-defined filling factors, the other when the scatterer connects two reservoirs. We illustrate its use by considering full counting statistics and the Fermi-edge singularity.
\end{abstract}

DOI: 10.1103/PhysRevB.77.165118

PACS number(s): 73.23.- b, 73.50.Td, 05.40.-a

\section{INTRODUCTION}

The pioneering works of Landauer ${ }^{1}$ and Büttiker ${ }^{2-4}$ lay the foundations for what is now known as the scattering approach to electron transport. The basic tenet is that a coherent conductor is characterized by its scattering matrix. More precisely, the transmission matrix defines a set of transparencies for the various channels or modes in which the electrons propagate through the conductor. As a consequence, conductance is the sum over transmission probabilities. Subsequently, it was discovered that the same transmission probabilities fully determine the current noise, also outside equilibrium, where the fluctuation-dissipation theorem does not hold. .,3 $^{5}$

Indeed, as the theory of full counting statistics ${ }^{6,7}$ (FCS) later revealed, the complete probability distribution for outcomes of a current measurement is entirely characterized by the transmission probabilities of the conductor. The fact that the scattering formalism gives such an elegant and complete description inspired some to revisit established results. Thus, for instance, interacting problems such as the Fermi-edge singularity ${ }^{8,9}$ (FES) was recast in the language of the scattering approach. ${ }^{10-13}$ The scattering approach has further been successfully employed in problems where a coherent conductor interacts with other elements, including, but not restricted to, measuring devices and an electromagnetic environment. ${ }^{14-17}$ It is also widely applied to study transport in mesoscopic superconductors. ${ }^{18}$

Many of these more advanced applications are unified through a method developed by Feynman and Vernon for characterizing the effect of one quantum system on another when they are coupled. ${ }^{19}$ The work of Feynman and Vernon dealt with the effect of a bath of oscillators coupled to a quantum system. It introduced the concept of a time contour describing a propagation first forward and then backward in time. By using the path-integral formalism, it was possible to characterize the bath by an "influence functional" that did not depend on the system that the bath was coupled to. This functional was treated nonperturbatively. A related development was due to Keldysh. ${ }^{20}$ While being a perturbative diagrammatic technique, it allowed for the treatment of general systems and shared the idea of a forward and backward time contour with Feynman and Vernon.
In general, the Feynman-Vernon method expresses the dynamics of a complex system in the form of an integral over a few fields $\chi(t)$. Each part of the system contributes to the integrand by a corresponding influence functional $\mathcal{Z}[\chi]$ or, synonymously, a Keldysh action $\mathcal{A}[\chi]=\ln \mathcal{Z}[\chi]$. Thus, the Keldysh action of a general scatterer can be used as a building block. In this way the action of a complicated nanostructure consisting of a network of scatterers can be constructed. As in the case of classical electronics, a simple set of rules, applied at the nodes of the network, suffice to describe the behavior of the whole network. ${ }^{21,22}$

The essential element of the approach is that the fields $\chi$ take different values on the forward and backward parts of the time contour. One writes this as $\chi_{ \pm}(t)$, where $+(-)$ corresponds to the forward (backward) part of the contour. The Keldysh action for a given subsystem is evaluated as a full nonlinear response of the subsystem to the fields $\chi_{ \pm}(t)$. [See Eq. (6) below for the precise mathematical definition.]

Applications involving the scattering approach require both the notion of the nonperturbative influence functional and the generality of Keldysh's formalism. Until now, the combination of the Feynman-Vernon method with the scattering approach was done on a case-specific basis: only those elements relevant to the particular application under consideration were developed. In this paper, we unify previous developments by deriving general formulas for the Keldysh action of a general scatterer connected to charge reservoirs.

The time-dependent fields $\chi_{+}(t)$ and $\chi_{-}(t)$ parametrize two Hamiltonians $\mathcal{H}_{+}(t)$ and $\mathcal{H}_{-}(t)$ that govern forward and backward evolutions in time, respectively. Since we are in the framework of the scattering approach, these field-dependent Hamiltonians are not the most natural objects to work with. Rather, depending on where the fields couple to the system, it is natural to incorporate their effect either in the scattering matrix of the conductor or in the Green's functions of the electrons in the reservoirs: the fields affecting the scattering potential inside the scatterer are incorporated in a timedependent scattering matrix. Since the fields $\chi_{ \pm}$for forward and backward evolutions are different, the scattering matrices for forward and backward evolutions differ. The effect of the fields perturbing the electrons far from the scatterer is incorporated in the time-dependent Green's functions of the 
electrons in distant reservoirs. A bias voltage field applied across a conductor can conveniently be ascribed to either Green's functions of the reservoirs or to a phase factor of the scattering matrix. The same holds for the counting fields encounterd in the theory of full counting statistics. There are, however, situations where our hand is forced. For instance, in the example of the Fermi-edge singularity, which we will discuss in Sec. VI, the time-dependent fields have to be incorporated in the scattering matrices.

Previous studies of the Keldysh action concentrate on situations where the fields $\chi_{ \pm}$can be incorporated in the reservoir Green's functions. These studies therefore assume stationary, contour-independent scattering matrices while allowing for a time dependence and/or time-contour dependence of the electron Green's functions. Early works (Refs. 23 and 24) used an action of this type to analyze Coulomb blockade phenomena. Later, the same action was understood in a wider context of arbitrary Green's functions. ${ }^{21,25}$ In this form, it has been used to treat problems involving, for example, interactions and superconductivity. The action employed in these studies corresponds to Eq. (4) and can readily be derived in the context of a nonlinear sigma model of disordered metals. ${ }^{26}$

The main innovation of the present work is to generalize the action to contour- and time-dependent scattering matrices. The only assumption we make is that scattering is instantaneous: we do not treat the delay time an electron spends inside the scattering region realistically.

The resulting scattering matrices associated with forward and backward evolutions are combined into one big matrix $\hat{s}$. It has a kernel $s\left(\alpha ; c, c^{\prime} ; t\right) \delta_{\alpha, \alpha^{\prime}} \delta\left(t-t^{\prime}\right)$, where the Keldysh indices $\alpha, \alpha^{\prime} \in\{+,-\}$ refer to the forward and backward parts of the time contour, $c, c^{\prime}$ are integers that refer to channel space, and $t, t^{\prime}$ are time indices that lie on the real line. The forward (backward) scattering matrix $\hat{s}_{+(-)}$with kernel $\hat{s}(\alpha$ $\left.=+(-) ; c, c^{\prime} ; t\right)$ obeys the usual unitarity condition $\hat{s}_{ \pm}^{\dagger} \hat{s}_{ \pm}=1$.

With the aide of these preliminary definitions, our main result is summarized by a formula for the Keldysh action.

$$
\mathcal{A}[\hat{s}]=\operatorname{Tr} \ln \left[\frac{1+\hat{G}}{2}+\hat{s} \frac{1-\hat{G}}{2}\right]-\operatorname{Tr} \ln \hat{s}_{-} .
$$

In this formula, $\hat{G}$ is the Keldysh Green's function characterizing the reservoirs connected to the scatterer. ${ }^{27}$ It is to be viewed as an operator with kernel $G\left(\alpha, \alpha^{\prime} ; c ; t, t^{\prime}\right) \delta_{c, c^{\prime}}$, where the indices carry the same meaning as in the definition of $\hat{s}$. This formula is completely general as demonstrated by the following:

(1) It holds for time-dependent scattering matrices that differ on the forward and backward time contours.

(2) It holds for multiterminal devices with more than two reservoirs.

(3) It holds for devices such as Hall bars, where particles in a single chiral channel enter and leave the conductor at different reservoirs.

(4) It holds when reservoirs cannot be characterized by stationary filling factors. Reservoirs may be superconducting or contain "counting fields," which couple them to a dynamical electromagnetic environment or a measuring device.
When the reservoirs can indeed be characterized by filling factors $\hat{f}(\varepsilon)$, the Keldysh structure can explicitly be traced out to yield

$$
\mathcal{A}\left[\hat{s}_{+}, \hat{s}_{-}\right]=\operatorname{Tr} \ln \left[\hat{s}_{-}(1-\hat{f})+\hat{s}_{+} \hat{f}\right]-\operatorname{Tr} \ln \hat{s}_{-} .
$$

In this expression, operators retain their channel structure and their time structure. In "time" representation, $\hat{f}$ is the Fourier transform to time of the reservoir filling factors and, as such, has a kernel $f\left(c ; t, t^{\prime}\right) \delta_{c, c^{\prime}}$ diagonal in channel space and depending on two times. In the stationary limit, this formula immediately reduces to the Levitov formula for lowfrequency FCS. ${ }^{7}$

Another formula that can be derived from Eq. (1) is valid for two terminal devices and a stationary, time-contourindependent scattering matrix but allows for arbitrary Green's functions in the terminals. Each terminal may still be connected to the scatterer by an arbitrary number of channels. We denote the two terminals left (L) and right (R). In this case, the reservoir Green's function has the form

$$
\hat{G}=\left(\begin{array}{cc}
\check{G}_{L} & 0 \\
0 & \check{G}_{R}
\end{array}\right)_{\text {channel space }},
$$

where $G_{L(R)}$ have no further channel space structure. The matrix structure in Keldysh and time indices (indicated by a check sign) is now retained in the trace, but the channel structure is traced out. Thus, the following is obtained:

$$
\mathcal{A}\left[\chi_{ \pm}\right]=\frac{1}{2} \sum_{n} \operatorname{Tr} \ln \left[1+T_{n} \frac{\left\{\check{G}_{L}\left[\chi_{ \pm}\right], \check{G}_{R}\left[\chi_{ \pm}\right]\right\}-2}{4}\right] .
$$

In this expression, the field dependence $\chi_{ \pm}$is shifted entirely to the Keldysh Green's functions $G_{L}$ and $G_{R}$ of the left and right reservoirs. This formula makes it explicit that the conductor is completely characterized by its transmission eigenvalues $T_{n}$.

The structure of the paper is as follows. After making the necessary definitions, we derive Eq. (1) from a model Hamiltonian. The derivation makes use of contour ordered Green's functions and the Keldysh technique. Subsequently, we derive the special cases of Eqs. (2) and (4).

We conclude by applying the formulas to several generic setups and verify that results agree with the existing literature. Particularly, we explain in detail how the present work is connected to the theory of full counting statistics and to the scattering theory of the Fermi-edge singularity.

\section{DERIVATION}

We consider a general scatterer connecting a set of charge reservoirs (Fig. 1). We allow the scatterer to be time dependent. A sufficient theoretical description is provided by a set of transport channels interrupted by a potential that causes interchannel scattering. We consider the regime where the scattering matrix is energy independent in the transport energy window. Since transport is purely determined by the scattering matrix, any model that produces the same scattering matrix gives identical results. Regardless of the actual 


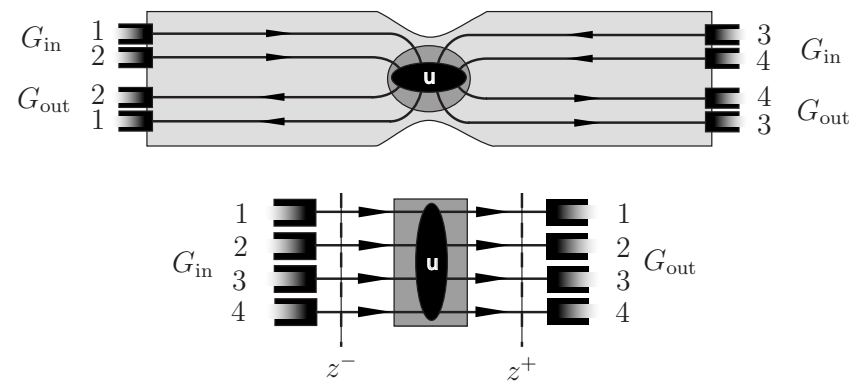

FIG. 1. We consider a general scatterer connected to reservoirs. The top figure is a diagram of one possible physical realization of a scatterer. Channels carry electrons toward and away from a scattering region (shaded dark gray), where interchannel scattering takes place. Reservoirs are characterized by Keldysh Green's functions $G_{\text {in(out). }}$ These Green's functions also carry a channel index in order to account for, among other things, voltage biasing. In setups such as the the quantum Hall experiment where there is a Hall voltage, $G_{\text {in }}$ will differ from $G_{\text {out }}$, while in an ordinary QPC, the two will be identical. The bottom figure shows how the physical setup is represented in our model. Channels are unfolded so that all electrons enter at $z^{-}$and leave at $z^{+}$.

microscopic detail, we may therefore conveniently take the Hamiltonian of the scatterer to be

$$
\mathcal{H}=v_{F} \sum_{m, n} \int d z \psi_{m}^{\dagger}(z)\left\{-i \delta_{m, n} \partial_{z}+u_{m, n}(z)\right\} \psi_{n}(z)+\mathcal{H}_{\mathrm{res}}+\mathcal{H}_{\mathrm{T}},
$$

where $\mathcal{H}_{\text {res }}$ represents the reservoirs, and $\mathcal{H}_{\mathrm{T}}$ takes account of tunneling between the conductor and the reservoirs. The scattering region and the reservoirs are spatially separated. This means that the scattering potential $u_{m n}(z)$ is nonzero only in a region $z^{-}<z<z^{+}$, while tunneling between the reservoirs and the conductor only takes place outside this region. Note that in our model, scattering channels have been "unfolded," so that instead of working with a channel that confines particles in the interval $(-\infty, 0]$ and allowing for propagation both in the positive and in the negative direction, we equivalently work with channels in which particles propagate along $(-\infty, \infty)$, but only in the positive direction. Hence, to make contact with most physical setups, we consider $-z$ and $z$ to refer to the same physical position in a channel but opposite propagation directions.

We consider the generating functional

$$
\begin{aligned}
\mathcal{Z}= & e^{\mathcal{A}}=\operatorname{Tr}\left[\mathcal{T}^{+} \exp \left\{-i \int_{t_{0}}^{t_{1}} d t \mathcal{H}^{+}(t)\right\}\right. \\
& \left.\times \rho_{0} \mathcal{T}^{-} \exp \left\{i \int_{t_{0}}^{t_{1}} d t \mathcal{H}^{-}(t)\right\}\right],
\end{aligned}
$$

in which $\mathcal{H}^{ \pm}$is obtained from $\mathcal{H}$ by replacing $u_{m n}(z)$ with arbitrary time-dependent functions $u_{m n}^{ \pm}(z, t)$. In this expressions, $\mathcal{T}^{+} \exp$ and $\mathcal{T}^{-}$exp respectively refer to time-ordered (i.e., largest time to the left) and anti-time-ordered (i.e., largest time to the right) exponentials. In the language of Feynman and Vernon, ${ }^{19}$ this is known as the influence functional.
It gives a complete characterization of the effect that the electrons in the scatterer have on any quantum system that they interact with. Furthermore, the functional $\mathcal{Z}$ generates expectation values of time-ordered products of operators as follows. Let $Q$ be an operator

$$
Q=\sum_{m n} \int_{z^{-}}^{z^{+}} d z \psi_{m}^{\dagger}(z) q_{m n}(z) \psi_{n}(z)
$$

Choose $u_{m n}^{ \pm}(z, t)=u_{m n}(z)+\chi_{ \pm}(t) q_{m n}(z)$. Then

$$
\begin{aligned}
\langle\mathcal{T} & \left.\left(\prod_{j=1}^{M} Q\left(t_{j}\right)\right) \mathcal{T}^{+}\left(\prod_{k=1}^{N} Q\left(t_{k}^{\prime}\right)\right)\right\rangle \\
= & \left.\prod_{j=1}^{M}\left(-i \frac{\delta}{\delta \chi^{-}\left(t_{j}\right)}\right) \prod_{k=1}^{N}\left(i \frac{\delta}{\delta \chi^{+}\left(t_{k}^{\prime}\right)}\right) \mathcal{Z}[\chi]\right|_{\chi=0} .
\end{aligned}
$$

By merging the power of the Keldysh formalism of contourordered Green's functions with that of the Landauer scattering formalism for quantum transport, we obtain an expression for $\mathcal{Z}$ in terms of the Keldysh Green's functions in the reservoirs and the time-dependent scattering matrices associated with $\hat{u}^{ \pm}(z, t)$.

The argument will proceed in the following steps:

(1) First, we introduce the key object that enables a systematic analysis of $\mathcal{Z}$, namely, the single particle Green's function $g$ of the conductor. We state the equations of motion that $g$ obeys.

(2) We define the Keldysh action $\mathcal{A}=\ln \mathcal{Z}$ and consider its variation $\delta \mathcal{A}$. We discover that $\delta \mathcal{A}$ can be expressed in terms of $g$.

(3) We therefore determine $g$ inside the scattering region in terms of the scattering matrix of the conductor and its value at the edges of the scattering region, where the reservoirs impose boundary conditions.

(4) This allows us to express the variation of the action in terms of the reservoir Green's functions $G_{\text {in(out) }}$ and the scattering matrix $s$ of the conductor.

(5) The variation $\delta \mathcal{A}$ is then integrated to find the action $\mathcal{A}$ and the generating functional $\mathcal{Z}$.

\section{A. Preliminaries: Definition of the Green's function}

The first step is to move from the Schrödinger picture to the Heisenberg picture. To shorten the notation, we define two time-evolution operators:

$$
\mathcal{U}_{ \pm}\left(t_{f}, t_{i}\right)=\mathcal{T}^{+} \exp \left\{-i \int_{t_{i}}^{t_{f}} d t^{\prime} \mathcal{H}^{ \pm}\left(t^{\prime}\right)\right\} .
$$

Associated with every Schrödinger picture operator, we define two Heisenberg operators, one corresponding to evolution with each of the two Hamiltonians $\mathcal{H}^{ \pm}$:

$$
Q_{ \pm}(t)=\mathcal{U}_{ \pm}\left(t_{f}, t_{i}\right)^{\dagger} Q \mathcal{U}_{ \pm}\left(t_{f}, t_{i}\right) .
$$

In order to have the tools of the Keldysh formalism at our disposal, we need to define four Green's functions as follows:

$$
\begin{aligned}
g_{m, n}^{++}\left(z, t ; z^{\prime}, t^{\prime}\right)= & -e^{\mathcal{A}} \operatorname{Tr}\left\{\mathcal{U}^{+}\left(t_{1}, t_{0}\right) \mathcal{T}^{+}\left[\psi_{n+}^{\dagger}\left(z^{\prime}, t^{\prime}\right) \psi_{m+}(z, t)\right]\right. \\
& \left.\times \rho_{0}\left[\mathcal{U}^{-}\left(t_{1}, t_{0}\right)\right]^{\dagger}\right\},
\end{aligned}
$$




$$
\begin{aligned}
g_{m, n}^{+-}\left(z, t ; z^{\prime}, t^{\prime}\right)= & e^{\mathcal{A}} \operatorname{Tr}\left\{\mathcal{U}^{+}\left(t_{1}, t_{0}\right) \psi_{m+}(z, t) \rho_{0} \psi_{n-}^{\dagger}\left(z^{\prime}, t^{\prime}\right)\right. \\
& \left.\times\left[\mathcal{U}^{-}\left(t_{1}, t_{0}\right)\right]^{\dagger}\right\}, \\
g_{m, n}^{-+}\left(z, t ; z^{\prime}, t^{\prime}\right)= & e^{\mathcal{A}} \operatorname{Tr}\left\{\mathcal{U}^{+}\left(t_{1}, t_{0}\right) \psi_{n+}^{\dagger}\left(z^{\prime}, t^{\prime}\right) \rho_{0} \psi_{m-}(z, t)\right. \\
& \left.\times\left[\mathcal{U}^{-}\left(t_{1}, t_{0}\right)\right]^{\dagger}\right\}, \\
g_{m, n}^{--}\left(z, t ; z^{\prime}, t^{\prime}\right)= & e^{\mathcal{A}} \operatorname{Tr}\left\{\mathcal{U}^{+}\left(t_{1}, t_{0}\right) \rho_{0} \mathcal{T}-\left[\psi_{n-}^{\dagger}\left(z^{\prime}, t^{\prime}\right) \psi_{m-}(z, t)\right]\right. \\
& \left.\times\left[\mathcal{U}^{-}\left(t_{1}, t_{0}\right)\right]^{\dagger}\right\} .
\end{aligned}
$$

Here, the symbol $\mathcal{T}^{+}$orders operators with larger time arguments to the left. If permutation is required to obtain the time-ordered form, the product is multiplied with $(-1)^{n}$, where $n$ is the parity of the permutation. Similarly, $\mathcal{T}^{-}$antitime-orders with the same permutation parity convention.

The Green's functions can be grouped into a matrix in Keldysh space as follows:

$$
g_{m, n}\left(z, t ; z^{\prime}, t^{\prime}\right)=\left(\begin{array}{ll}
g_{m, n}^{++}\left(z, t ; z^{\prime}, t^{\prime}\right) & g_{m, n}^{+-}\left(z, t ; z^{\prime}, t^{\prime}\right) \\
g_{m, n}^{-+}\left(z, t ; z^{\prime}, t^{\prime}\right) & g_{m, n}^{--}\left(z, t ; z^{\prime}, t^{\prime}\right)
\end{array}\right) .
$$

Notation can be further shortened by incorporating channel indices into the matrix structure of the Green's function, thereby defining an object $\bar{g}\left(z, t ; z^{\prime}, t^{\prime}\right)$. The element of $\bar{g}$ that is located on row $m$ and column $n$ is the $2 \times 2$ matrix $g_{m, n}$.

The Green's function satisfies the equation of motion

$$
\begin{gathered}
\left\{i \partial_{t}+v_{F} i \partial_{z}-v_{F} \bar{u}(z, t)\right\} \bar{g}\left(z, t ; z^{\prime}, t^{\prime}\right)-\int d t^{\prime \prime} \Sigma(z ; t \\
\left.-t^{\prime \prime}\right) \bar{g}\left(z, t^{\prime \prime} ; z^{\prime} t^{\prime}\right)=\delta\left(t-t^{\prime}\right) \delta\left(z-z^{\prime}\right) \overline{1} .
\end{gathered}
$$

The delta functions on the right of Eq. (13) encode the fact that due to time ordering, $g_{m n}^{++}$and $g_{m n}^{--}$have a step structure,

$$
\frac{1}{v_{F}} \theta\left(z-z^{\prime}\right) \delta\left(t-t^{\prime}-\frac{z-z^{\prime}}{v_{F}}\right) \delta_{m n}+f\left(z, t ; z^{\prime} t^{\prime}\right),
$$

where $f$ is continuous in all its arguments. The self-energy

$$
\Sigma(z ; \tau)=-i \frac{\bar{G}_{\text {in }}(\tau)}{2 \tau_{c}} \theta\left(z^{-}-z\right)-i \frac{\bar{G}_{\text {out }}(\tau)}{2 \tau_{c}} \theta\left(z-z^{+}\right)
$$

results from the reservoirs and determines how the scattering channels are filled. It is a matrix in Keldysh space. The time $\tau_{c}$ is the characteristic time that correlations survive in the region of the conductor that is connected to the reservoirs before the reservoirs scramble them. $\bar{G}_{\text {in(out) }}(\tau)$ is the reservoir Green's functions, where electrons enter (leave) the scattering region, summed over reservoir levels, and normal- ized to be dimensionless. This form of the self-energy can be derived from the following model for the reservoirs: we imagine every point $z$ in a channel $m$ outside $\left(z^{-}, z^{+}\right)$to exchange electrons with an independent Fermion bath with a constant density of states $\nu$. The terms $\mathcal{H}_{\text {res }}$ and $\mathcal{H}_{\mathrm{T}}$ are explicitly

$$
\begin{gathered}
\mathcal{H}_{\mathrm{res}}=\sum_{m} \int d E \nu \int_{z \in\left(z^{-}, z^{+}\right)} d z E a_{m}^{\dagger}(E, z) a_{m}(E, z), \\
\mathcal{H}_{\mathrm{T}}=\sum_{m} c_{m} \int d E \nu \int_{z \in\left(z^{-}, z^{+}\right)} d z \psi_{m}^{\dagger}(z) a_{m}(E, z)+a_{m}^{\dagger}(E, z) \psi_{m}(z),
\end{gathered}
$$

where the tunneling amplitude $c_{m}$ characterizes the coupling between the reservoir and the channel $m$. More general reservoir models need not be considered, since, as we shall see shortly, the effect of the reservoirs is entirely contained in boundary conditions on the Green's function $\bar{g}$ inside the scatterer. This boundary condition does not depend on microscopic detail, but only on the reservoir Green's functions $\bar{G}_{\text {in(out) }}$.

We do not need to know the explicit form of the reservoir Green's functions yet. Rather, the argument below exclusively relies on the property of $\bar{G}_{\text {in(out) }}$ that it squares to unity: ${ }^{27}$

$$
\int d t^{\prime \prime} \bar{G}\left(t-t^{\prime \prime}\right)_{\mathrm{in}(\mathrm{out})} \bar{G}\left(t^{\prime \prime}-t^{\prime}\right)_{\mathrm{in}(\mathrm{out})}=\delta\left(t-t^{\prime}\right) \overline{1} .
$$

A differential equation similar to Eq. (13) holds for $\bar{g}^{\dagger}$.

\section{B. Varying the action $\mathcal{A}$}

We are now ready to attack the generating functional $\mathcal{Z}$. For our purposes, it is most convenient to consider $\mathcal{A}=\ln \mathcal{Z}$. We will call this object the action. Our strategy is as follows: we will obtain an expression for the variation $\delta \mathcal{A}$ resulting from a variation $\hat{u}(z, t) \rightarrow \hat{u}(z, t)+\delta \hat{u}(z, t)$ of the scattering potentials. This expression will be in terms of the reservoir filling factors $\hat{f}$ and the scattering matrices associated with $\hat{u}(z, t)$. We then integrate to find $\mathcal{A}$.

We start by writing

$$
\begin{aligned}
\delta \mathcal{A}= & -i v_{F} e^{\mathcal{A}} \sum_{m, n} \int_{t_{0}}^{t_{1}} d t \int d z\left[\delta u_{n, m}^{+}(z, t)\left\langle\psi_{m}^{\dagger}(z) \psi_{n}(z)\right\rangle_{+}(t)\right. \\
& \left.-\delta u_{n, m}^{-}(z, t)\left\langle\psi_{m}^{\dagger}(z) \psi_{n}(z)\right\rangle_{-}(t)\right]
\end{aligned}
$$

where

$$
\left\langle\psi_{m}^{\dagger}(z) \psi_{n}(z)\right\rangle_{+}(t)=\operatorname{Tr}\left[\mathcal{T}^{+} \exp \left\{-i \int_{t}^{t_{1}} d t^{\prime} \mathcal{H}^{+}\left(t^{\prime}\right)\right\} \psi_{m}^{\dagger}(z) \psi_{n}(z) \mathcal{T}^{+} \exp \left\{-i \int_{t_{0}}^{t} d t^{\prime} \mathcal{H}^{+}\left(t^{\prime}\right)\right\} \rho_{0} \mathcal{T}^{-} \exp \left\{i \int_{t_{0}}^{t_{1}} d t^{\prime} \mathcal{H}^{-}\left(t^{\prime}\right)\right\}\right],
$$




$$
\left\langle\psi_{m}^{\dagger}(z) \psi_{n}(z)\right\rangle_{-}(t)=\operatorname{Tr}\left[\mathcal{T}^{+} \exp \left\{-i \int_{t_{0}}^{t_{1}} d t^{\prime} \mathcal{H}^{+}\left(t^{\prime}\right)\right\} \rho_{0} \mathcal{T}^{-} \exp \left\{i \int_{t_{0}}^{t} d t^{\prime} \mathcal{H}^{-}\left(t^{\prime}\right)\right\} \psi_{m}^{\dagger}(z) \psi_{n}(z) \mathcal{T}^{-} \exp \left\{-i \int_{t}^{t_{1}} d t^{\prime} \mathcal{H}^{-}\left(t^{\prime}\right)\right\}\right] .
$$

\section{Expressing $\delta \mathcal{A}$ in terms of the Green's function $g$}

In terms of the defined Green's functions, the variation $\delta \mathcal{A}$ becomes

$$
\begin{aligned}
\delta \mathcal{A}= & i v_{F} \sum_{m, n} \int_{t_{0}}^{t_{1}} d t \int d z\left[\delta u_{n, m}^{+}(z, t) g_{m, n}^{++}\left(z, t-0^{+} ; z, t\right)\right. \\
& \left.+\delta u_{n, m}^{-}(z, t) g_{m, n}^{--}\left(z, t+0^{+} ; z, t\right)\right] \\
= & i v_{F} \int_{t_{0}}^{t_{1}} d t \int d z \operatorname{Tr}\left[\delta \bar{u}(z, t) \bar{g}\left(z, t+0^{k} ; z, t\right)\right] .
\end{aligned}
$$

The object $\delta \bar{u}$ is constructed by combining the channel and Keldysh indices of the variation of the potential. The trace is over both Keldysh and channel indices. The symbol $0^{k}$ refers to the regularization explicitly indicated in the first line, i.e., the first time argument of $g^{++}\left(z, t-0^{+} ; z, t\right)$ is evaluated an infinitesimal time $0^{+}>0$ before the second argument, while in $g^{--}\left(z, t+0^{-} ; z, t\right)$, the first time argument is evaluated an infinitesimal time $0^{+}$after the second. This is done so that the time ordering (anti-time-ordering) operations give the order of creation and annihilation operators required in Eq. (18).

It proves very inconvenient to deal with the $0^{k}$ regularization of Eq. (20). It is preferable to have the first time arguments of both $g^{++}$and $g^{--}$evaluated an infinitesimal time $0^{+}$ before the second. Taking into account the step structure of $\hat{g}^{++}$, we have

$$
\begin{aligned}
\bar{g}\left(z, t+0^{k} ; z^{\prime} t^{\prime}\right)= & \bar{g}\left(z, t-0^{+} ; z^{\prime}, t^{\prime}\right) \\
& +\frac{1}{v_{F}} \delta\left(t-t^{\prime}-\frac{z-z^{\prime}}{v_{F}}\right) \hat{1}\left(\frac{1-\check{\tau}_{3}}{2}\right) .
\end{aligned}
$$

Here, $\tau_{3}$ is the third Pauli matrix

$$
\left(\begin{array}{cc}
1 & 0 \\
0 & -1
\end{array}\right)
$$

acting in Keldysh space. The equations of motion allow us to relate $\bar{g}\left(z, t-0^{+} ; z^{\prime}, t^{\prime}\right)$ for points $z$ and $z^{\prime}$ inside the scattering region where $\bar{u}$ is nonzero to the value of $\bar{g}$ at $z^{-}$where electrons enter the scatterer. For $z \leqslant z^{\prime}$ and $t \leqslant t^{\prime}$, the equations of motion give

$$
\begin{aligned}
& \bar{g}\left(z, t+\frac{z-z^{-}}{v_{F}}-0 ; z^{\prime}, t+\frac{z^{\prime}-z^{-}}{v_{F}}\right) \\
& \quad=\bar{s}(z, t) \bar{g}\left(z^{-}, t-0^{+} ; z^{\prime}, t^{\prime}\right) \bar{s}^{\dagger}\left(z^{\prime}, t^{\prime}\right),
\end{aligned}
$$

where

$$
\bar{s}(z, t)=\mathcal{Z} \exp \left\{-i \int_{z^{-}}^{z} d z^{\prime \prime} \bar{u}\left(z^{\prime \prime}, t+\frac{z^{\prime \prime}-z^{-}}{v_{F}}\right)\right\} .
$$

The symbol $\mathcal{Z}$ indicates that the exponent is ordered along the $z$ axis, with the largest coordinate in the integrand to the left. Note that the potential $\bar{u}$ at position $z$ is evaluated at the time instant $t+\left(z-z^{-}\right) / v_{F}$ that an electron entering the scattering region at time $t$ reaches $z$. Often the time dependence of the potential is slow on the time scale $\left(z^{+}-z^{-}\right) / v_{F}$, representing the time a transported electron spends in the scattering region, and $\bar{u}\left(z, t+\frac{z-z^{-}}{v_{F}}\right)$ can be replaced with $\bar{u}(z, t)$. This is, however, not required for the analysis that follows to be valid.

Substitution into Eq. (24) yields

$$
\begin{aligned}
\delta A= & v_{F} \int d t \operatorname{Tr}\left[\bar{w}(t) g\left(z^{-}, t-0^{+} ; z^{-}, t\right)\right] \\
& -\int d t \lim _{t^{\prime} \rightarrow t} \delta\left(t-t^{\prime}\right) \operatorname{Tr}\left[\bar{w}(t) \hat{1}\left(\frac{1-\check{\tau}_{3}}{2}\right)\right],
\end{aligned}
$$

with

$$
\bar{w}(t)=-i \int_{z^{-}}^{z^{+}} d z \bar{s}^{\dagger}(z, t) \delta \bar{u}\left(z, t+\frac{z-z^{-}}{v_{F}}\right) \bar{s}(z, t)=\bar{s}^{\dagger}(t) \delta \bar{s}(t) .
$$

In this equation, $z^{+}$is located where electrons leave the scatterer. Importantly, here, Tr still denotes a trace over channel and Keldysh indices. We will later on redefine the symbol to include also a trace over the (continuous) time index, at which point the second term in Eq. (24) will (perhaps deceptively) look less offensive, but not yet. In the last line of Eq. (25), $\bar{s}(t)=\bar{s}\left(z^{+}, t\right)$ is the (time-dependent) scattering matrix. We sent the boundaries $t_{0}$ and $t_{1}$, over which we integrate in the definition of the action, to $-\infty$ and $\infty$, respectively, which will allow us to Fourier transform to frequency in a moment. The action remains well defined as long as the potentials $u^{+}$ and $u^{-}$only differ for a finite time.

\section{Relating $g$ inside the scattering region to $g$ at reservoirs: Imposing boundary conditions implied by reservoirs}

Our task is now to find $\bar{g}\left(z^{-}, t-0^{+} ; z^{-}, t\right)$. Because of the $t-t^{\prime}$ dependence of the self-energy, it is convenient to transform to Fourier space, where

$$
\bar{g}\left(z, \varepsilon ; z^{-}, \varepsilon^{\prime}\right)=\int d t d t^{\prime} e^{i \varepsilon t} \bar{g}\left(z, t ; z^{-}, t^{\prime}\right) e^{-i \varepsilon^{\prime} t^{\prime}},
$$




$$
\bar{G}_{\text {in (out) }}(\varepsilon)=\int d t e^{i \varepsilon t} \bar{G}(t)_{\text {in (out) }}
$$

In frequency domain, the property that $\bar{G}_{\text {in(out) }}$ squares to unity is expressed as $\bar{G}_{\text {in(out) }}(\varepsilon)^{2}=\overline{1}$. [Due to the standard conventions for Fourier transforms, the matrix elements of the identity operator in energy domain is $2 \pi \delta\left(\varepsilon-\varepsilon^{\prime}\right)$.] The equation of motion for $z<z^{-}$reads

$$
\left\{-i \varepsilon+v_{F} \partial_{z}+\frac{\bar{G}_{\text {in }}(\varepsilon)}{2 \tau_{c}}\right\} \bar{g}\left(z, \varepsilon ; z^{-}, \varepsilon^{\prime}\right)=0 .
$$

There is no inhomogeneous term on the right-hand side because we restrict $z$ to be less than $z^{-}$. We thus find

$$
\begin{aligned}
\bar{g}\left(z^{-}-0^{+}, \varepsilon ; z^{-}, \varepsilon^{\prime}\right)= & e^{i \varepsilon \Delta z / v_{F}} \exp \left[-\frac{\bar{G}_{\text {in }}(\varepsilon)}{2 l_{c}} \Delta z\right] \\
& \times \bar{g}\left(z^{-}-\Delta z, \varepsilon ; z^{-}, \varepsilon^{\prime}\right) .
\end{aligned}
$$

Here, the correlation length $l_{c}$ is the correlation time $\tau_{c}$ multiplied by the Fermi velocity $v_{F}$. By using the fact that $\bar{G}(\varepsilon)_{\text {in }}$ squares to unity, it is easy to verify that

$$
\begin{aligned}
\exp \left\{-\frac{\bar{G}_{\mathrm{in}}(\varepsilon)}{2 l_{c}} \Delta z\right\}= & \frac{1+\bar{G}_{\mathrm{in}}(\varepsilon)}{2} \exp \left(-\frac{\Delta z}{2 l_{c}}\right) \\
& +\frac{1-\bar{G}_{\mathrm{in}}(\varepsilon)}{2} \exp \left(\frac{\Delta z}{2 l_{c}}\right)
\end{aligned}
$$

Since spacial correlations decay beyond $z^{-}, \quad \bar{g}\left(z^{-}\right.$ $\left.-\Delta z, \varepsilon ; z^{-}, \varepsilon^{\prime}\right)$ does not blow up as we make $\Delta z$ larger. From this we derive the condition

$$
\left[1+\bar{G}_{\text {in }}(\varepsilon)\right] \bar{g}\left(z^{-}-0^{+}, \varepsilon ; z^{-}, \varepsilon^{\prime}\right)=0 .
$$

Transformed back to the time domain, this reads

$$
\int d t^{\prime \prime}\left[\delta\left(t-t^{\prime \prime}\right)+\bar{G}_{\text {in }}\left(t-t^{\prime \prime}\right)\right] \bar{g}\left(z^{-}-0^{+}, t^{\prime \prime} ; z^{-}, t^{\prime}\right)=0 .
$$

We can play the same game at $z^{+}$, where particles leave the scatterer. The equation of motion reads

$$
\begin{aligned}
& \left\{-i \varepsilon+v_{F} \partial_{z}+\theta\left(z-z^{+}\right) \frac{\bar{G}_{\text {out }}(\varepsilon)}{2 \tau_{c}}\right\} \bar{g}\left(z, \varepsilon ; z^{+}, \varepsilon^{\prime}\right) \\
& =2 \pi \delta\left(z-z^{\prime}\right) \delta\left(\varepsilon-\varepsilon^{\prime}\right) .
\end{aligned}
$$

This has the general solution

$$
\begin{aligned}
\bar{g}\left(z, \varepsilon ; z^{\prime}, \varepsilon^{\prime}\right)= & \exp \left\{i \varepsilon \frac{z-z^{\prime}}{v_{F}}-\left[\left(z-z^{+}\right) \theta\left(z-z^{+}\right)-\left(z^{\prime}-z^{+}\right)\right.\right. \\
& \left.\left.\times \theta\left(z^{\prime}-z^{+}\right)\right] \frac{\bar{G}_{\text {out }}(\varepsilon)}{2 l_{c}}\right\}\left[\bar{g}\left(z^{\prime}-0^{+}, \varepsilon^{\prime} ; z^{\prime}, \varepsilon^{\prime}\right)\right. \\
& \left.+\frac{2 \pi}{v_{F}} \theta\left(z-z^{\prime}\right) \delta\left(\varepsilon-\varepsilon^{\prime}\right)\right]
\end{aligned}
$$

We will need to relate the Green's function evaluated at $z$ $\left\langle z^{+}\right.$to the Green's function evaluated at $z>z^{+}$, and so we explicitly show the inhomogeneous term. The same kind of argument employed at $z^{-}$then yields the condition

$$
\left[1-\bar{G}_{\text {out }}(\varepsilon)\right]\left[\bar{g}\left(z^{+}-0^{+}, \varepsilon ; z^{+}, \varepsilon^{\prime}\right)+\frac{2 \pi}{v_{F}} \delta\left(\varepsilon-\varepsilon^{\prime}\right)\right]=0,
$$

where the inhomogeneous term in the equation of motion is responsible for the delta function. In time domain, this reads

$$
\begin{gathered}
\int d t^{\prime \prime}\left[\delta\left(t-t^{\prime \prime}\right)-\bar{G}_{\text {out }}\left(t-t^{\prime \prime}\right)\right]\left[\bar{g}\left(z^{+}-0^{+}, t^{\prime \prime} ; z^{+}, t^{\prime}\right)\right. \\
\left.+\frac{1}{v_{F}} \delta\left(t^{\prime \prime}-t^{\prime}\right)\right]=0 .
\end{gathered}
$$

It remains for us to relate $\bar{g}\left(z^{+}-0^{+}, t+\frac{z^{+}-z^{-}}{v_{F}} ; z^{+}, t^{\prime}+\frac{z^{+}-z^{-}}{v_{F}}\right)$ to $\bar{g}\left(z^{-}-0^{+}, t ; z^{-}, t^{\prime}\right)$. This is done with the help of Eq. (22), from which follows

$$
\begin{gathered}
\bar{g}\left(z^{+}-0^{+}, t+\frac{z^{+}-z^{-}}{v_{F}} ; z^{+}, t^{\prime}+\frac{z^{+}-z^{-}}{v_{F}}\right) \\
=\bar{s}(t) \bar{g}\left(z^{-}-0^{+}, t ; z^{-}, t^{\prime}\right) \bar{s}^{\dagger}\left(t^{\prime}\right) .
\end{gathered}
$$

We substitute this into Eq. (35), multiply from the right with $\bar{s}\left(t^{\prime}\right)$ and from the left with $\bar{s}^{\dagger}(t)$. If we define $\bar{G}_{\text {out }}^{\prime}\left(t, t^{\prime}\right)$ $=\bar{s}^{\dagger}(t) \bar{G}_{\text {out }}\left(t-t^{\prime}\right) \bar{s}\left(t^{\prime}\right)$, the resulting boundary condition is

$$
\begin{gathered}
\int d t^{\prime \prime}\left[\delta\left(t-t^{\prime \prime}\right)-\bar{G}_{\text {out }}^{\prime}\left(t-t^{\prime \prime}\right)\right]\left[\bar{g}\left(z^{-}-0^{+}, t^{\prime \prime} ; z^{-}, t^{\prime}\right)\right. \\
\left.+\frac{1}{v_{F}} \delta\left(t^{\prime \prime}-t^{\prime}\right)\right]=0 .
\end{gathered}
$$

\section{E. Finding the variation of the action in terms of the reservoir Green's functions and the scattering matrix}

At this point, it is convenient to incorporate time into the matrix structure of the objects $\bar{G}_{\mathrm{in}}, \bar{G}_{\text {out }}^{\prime}$, and $\bar{g}$. The resulting matrices will be written without overbars. Thus, for instance, $s$ will denote a matrix diagonal in time indices, whose entry $\left(t, t^{\prime}\right)$ is $\delta\left(t-t^{\prime}\right) \bar{s}(t)$. Similarly, the $\left(t, t^{\prime}\right)$ entry of $G_{\text {in(out) }}$ is $\bar{G}_{\text {in(out) }}\left(t-t^{\prime}\right)$. Also, let $g^{-}$be the matrix whose $\left(t, t^{\prime}\right)$ entry is $\bar{g}\left(z^{-}-0^{+}, t ; z^{-}, t^{\prime}\right)$. In this notation, $G_{\text {in }}^{2}=G_{\text {out }}^{\prime 2}=I$ and Eqs. (31) and (37) read

$$
\begin{gathered}
\left(I+G_{\text {in }}\right) g^{-}=0, \\
\left(I-G_{\text {out }}^{\prime}\right)\left(g^{-}+1 / v_{F}\right)=0 .
\end{gathered}
$$

These two equations uniquely determine $g^{-}$as follows: from the first of the two equations, we have

$$
0=G_{\text {out }}^{\prime}\left(I+G_{\text {in }}\right) g^{-}=-\left(I-G_{\text {out }}^{\prime}\right) g^{-}+\left(I+G_{\text {out }}^{\prime} G_{\text {in }}\right) g^{-} .
$$

In the first term, we can make the substitution $-\left(I-G_{\text {out }}^{\prime}\right) g^{-}$ $=\left(I-G_{\text {out }}^{\prime}\right) / v_{F}$, which follows from Eq. (38). Thus, we find 


$$
g^{-}=-\frac{1}{v_{F}} \frac{1}{I+G_{\text {out }}^{\prime} G_{\text {in }}}\left(I-G_{\text {out }}^{\prime}\right)=\frac{1}{v_{F}}\left(1-G_{\text {in }}\right) \frac{1}{G_{\text {out }}^{\prime}+G_{\text {in }}}
$$

and the last line follows from the fact that $G_{\text {in }}^{2}=G_{\text {out }}^{\prime 2}=I$. We have taken special care here to allow for different reservoir Green's functions at $z^{-}$, where particles enter the conductor, and at $z^{+}$, where they leave the conductor. In order to proceed, we must now absorb the difference between the two Green's functions in the scattering matrix. We define $\Lambda$ through the equation

$$
\bar{G}_{\text {out }}=\Lambda^{-1} G_{\text {in }} \Lambda
$$

and drop the subscripts on the Green's functions by setting $G \equiv G_{\text {in }}$. Substituting back into Eq. (24) for the variation of the action yields

$$
\delta \mathcal{A}=\operatorname{Tr}\left[\delta s^{\prime}(1-G) \frac{1}{G s^{\prime}+s^{\prime} G}\right]-\operatorname{Tr}\left[\delta \hat{s}_{-}\left(\hat{s}_{-}\right)^{\dagger}\right],
$$

where the trace is over time, channel, and, in the first term, Keldysh indices. The operator $s^{\prime}$ is related to the scattering matrix $s$ through $s^{\prime}=\Lambda s$.

\section{F. Integrating the variation to find the action $\mathcal{A}$}

We now have to integrate $\delta \mathcal{A}$ to find $\mathcal{A}$. This is most conveniently done by working in a basis where $G$ is diagonal. Since $G^{2}=1$, every eigenvalue of $G$ is \pm 1 . Therefore, there is a basis in which

$$
G=\left(\begin{array}{cc}
I & 0 \\
0 & -I
\end{array}\right)
$$

In this representation, $s^{\prime}$ can be written as

$$
s^{\prime}=\left(\begin{array}{cc}
s_{11}^{\prime} & s_{12}^{\prime} \\
s_{21}^{\prime} & s_{22}^{\prime}
\end{array}\right) \text {. }
$$

Here, the two indices of the subscript have the following meaning: the first refers to a left eigenspace of $G$, the second to a right eigenspace. The subscript 1 denotes the subspace of eigenstates of $G$ with eigenvalue 1 . The subscript 2 refers to the subspace of eigenstates of $G$ with eigenvalue -1 . In this representation,

$$
(1-G) \frac{1}{G s^{\prime}+s^{\prime} G}=\left(\begin{array}{cc}
0 & 0 \\
0 & \left(s_{22}^{\prime}\right)^{-1}
\end{array}\right),
$$

so that

$$
\delta \mathcal{A}=\operatorname{Tr}\left[\delta s_{22}^{\prime}\left(s_{22}^{-1}\right)^{\prime}\right]-\operatorname{Tr}\left[\delta \hat{s}_{-}\left(\hat{s}_{-}\right)^{\dagger}\right],
$$

and thus

$$
\begin{aligned}
& \mathcal{A}=\operatorname{Tr} \ln s_{22}^{\prime}-\operatorname{Tr} \ln s_{-}, \\
& e^{\mathcal{A}}=\left(\operatorname{Det} s_{-}\right)^{-1} \operatorname{Det} s_{22}^{\prime} .
\end{aligned}
$$

In these equations, $s_{-}$is the scattering matrix associated with $\mathcal{H}^{-}$as defined previously. Its time structure is to be included in the operations of taking the trace and determinant.
Note that in the representation where $G$ is diagonal, the following holds:

$$
\frac{1+G}{2}+s^{\prime} \frac{1-G}{2}=\left(\begin{array}{cc}
I & s_{12}^{\prime} \\
0 & s_{22}^{\prime}
\end{array}\right)
$$

Due to the upper-(block)-triangular structure, it holds that Det $s_{22}^{\prime}=\operatorname{Det}\left[\frac{1+G}{2}+s^{\prime} \frac{1-G}{2}\right]$, leading to our main result

$$
\mathcal{A}=\operatorname{Tr} \ln \left[\frac{1+G}{2}+s^{\prime} \frac{1-G}{2}\right]-\operatorname{Tr} \ln s_{-},
$$

where it has to be noted that many matrices have the same determinant as the above. Some obvious examples include

$$
\begin{gathered}
\left(\begin{array}{cc}
I & 0 \\
0 & s_{22}^{\prime}
\end{array}\right)=(1+G) / 2+(1-G) s^{\prime}(1-G) / 4, \\
\left(\begin{array}{cc}
I & 0 \\
s_{21}^{\prime} & s_{22}^{\prime}
\end{array}\right)=(1+G) / 2+(1-G) s^{\prime} / 2 .
\end{gathered}
$$

\section{TRACING OUT THE KELDYSH STRUCTURE}

Up to this point, the only property of $G$ that we relied on was the fact that it squares to identity. Hence, the result [Eq. (49)] holds in a setting that is more general than that of a scatterer connected to reservoirs characterized by filling factors. (The reservoirs may, for instance, be superconducting.) In the specific case of reservoirs characterized by filling factors, the following holds:

$$
\bar{G}(\tau)=\int \frac{d \varepsilon}{2 \pi} e^{-i \varepsilon \tau}\left(\begin{array}{ll}
1-2 \hat{f}(\varepsilon) & 2 \hat{f}(\varepsilon) \\
2-2 \hat{f}(\varepsilon) & -1+2 \hat{f}(\varepsilon)
\end{array}\right) .
$$

Here, $\hat{f}(\epsilon)$ is the diagonal in channel indices and $f_{m}(\epsilon)$ is the filling factor in the reservoir connected to channel $m$. We will also assume that electrons enter and leave a channel from the same reservoir, so that $G_{\text {in }}=G_{\text {out }}$ and, hence, $s^{\prime}=s$. We recall as well that the Keldysh structure of the scattering matrix is

$$
s=\left(\begin{array}{cc}
\hat{s}_{+} & 0 \\
0 & \hat{s}_{-}
\end{array}\right)
$$

Here, $\hat{s}_{ \pm}$have channel and time (or, equivalently, energy) indices. $\hat{s}_{ \pm}$is diagonal in time indices, with the entries on the time diagonal the time-dependent scattering matrices corresponding to an evolution with the Hamiltonians $\mathcal{H}_{ \pm}$.

With this structure in Keldysh space, we find

$$
e^{\mathcal{A}}=\operatorname{Det}\left(\begin{array}{cc}
1+\left(\hat{s}_{+}-1\right) \hat{f} & -\left(\hat{s}_{+}-1\right) \hat{f} \\
\left(\hat{s}_{-}-1\right)(\hat{f}-1) & \hat{s}_{-}(1-\hat{f})+\hat{f}
\end{array}\right) \operatorname{Det}\left(\begin{array}{ll}
1 & \\
& \hat{s}_{-}^{-1}
\end{array}\right) \text {. }
$$

We can remove the Keldysh structure from the determinant with the aid of the general formula 


$$
\operatorname{Det}\left(\begin{array}{ll}
A & B \\
C & D
\end{array}\right)=\operatorname{Det}\left(A D-A C A^{-1} B\right)=\operatorname{Det}\left(D A-C A^{-1} B A\right) \text {. }
$$

Noting that in our case the matrices $B$ and $A$ commute, so that $C A^{-1} B A=C B$, we have

$$
\begin{aligned}
e^{\mathcal{A}}= & \operatorname{Det}\left\{\left[\hat{s}_{-}(1-\hat{f})+\hat{f}\right][1+(\hat{s}-1) \hat{f}]-\left[\hat{s}_{-}(1-\hat{f})+\hat{f}-1\right]\right. \\
& \left.\times\left(\hat{s}_{+}-1\right) \hat{f}\right\} \operatorname{Det}\left(\hat{s}_{-}^{-1}\right)=\operatorname{Det}\left[\hat{s}_{-}(1-\hat{f})+\hat{s}_{+} \hat{f}\right] \operatorname{Det}\left(\hat{s}_{-}^{-1}\right) .
\end{aligned}
$$

\section{AN EXAMPLE: FULL COUNTING STATISTICS OF TRANSPORTED CHARGE}

A determinant formula of this type appears in the literature of full counting statistics ${ }^{7}$ of transported charge. This formula can be stated as follows: the generating function for transported charge through a conductor characterized by a time-independent scattering matrix $\hat{s}$ is

$$
\mathcal{Z}(\chi)=\operatorname{Det}\left[1+\left(\hat{s}_{-\chi}^{\dagger} \hat{s}_{\chi}-1\right) \hat{f}\right],
$$

where $\hat{s}_{\chi}$ is a scattering matrix, modified to depend on the counting field $\chi$ that, in this case, is time independent. (The precise definition may be found below.)

As a consistency check of our results, we apply our analysis to rederive this formula. We will consider the most general setup, where every scattering channel is connected to a distinct voltage-biased terminal. To address the situation where leads connect several channels to the same terminal, the voltages and counting fields associated with channels in the same lead are set equal.

The full counting statistics of charge transported through a scatterer in a time interval $t$ is defined as

$$
\mathcal{Z}(\chi, t)=\left\langle e^{i \mathcal{H}_{\chi} t} e^{-i \mathcal{H}_{-} x^{t}}\right\rangle .
$$

In this equation, the Hamiltonian $\mathcal{H}_{\chi}$ is given by

$$
\begin{aligned}
\mathcal{H}_{\chi}= & v_{f} \sum_{m, n} \int d z \psi_{m}^{\dagger}(z)\left\{-i \partial_{z} \delta_{m, n}+u_{m, n}(z)\right\} \psi_{n}(z) \\
& +\sum_{m} \chi_{m} I_{m}\left(z_{0}\right)
\end{aligned}
$$

where $I_{m}\left(z_{0}\right)$ is the current in channel $m$ at the point $z_{0}$, which is taken to lie outside the scattering region. The full counting statistics is thus generated by coupling the counting field $\chi_{m}$ to the current operator in a channel $m$.

Explicitly, the current operator in channel $m$ is given by

$$
I_{m}\left(z_{0}\right)=v_{F}\left[\psi_{m}^{\dagger}\left(z_{0}\right) \psi_{m}\left(z_{0}\right)-\psi_{m}^{\dagger}\left(-z_{0}\right) \psi_{m}\left(-z_{0}\right)\right] .
$$

To understand this equation, recall that the coordinates $z_{0}$ and $-z_{0}$ in channel $m$ refer to the same point in space, but opposite propagation directions.

The presence of current operators in Eq. (58) can be incorporated in the potential by defining a transformed potential

$$
u_{m, n}^{(\chi)}(z)=u_{m, n}(z)+\delta_{m, n} \frac{\chi_{m}}{2}\left[\delta\left(z-z_{0}\right)-\delta\left(z+z_{0}\right)\right] .
$$

Introducing counting fields that transform $\mathcal{H}_{0} \rightarrow \mathcal{H}_{\chi}$ is thus achieved by transforming $u \rightarrow u^{(\chi)}$.

The calculation of the full counting statistics has now been cast into the form of the trace of a density matrix after forward and backward time evolutions controlled by different scattering potentials. Our result [Eq. (55)] is therefore applicable, with

$$
\hat{s}_{ \pm}=\mathcal{Z} \exp \left(-i \int_{z_{-}}^{z_{+}} d z \hat{u}^{( \pm \chi)}(z)\right)=e^{\mp i \hat{\chi} / 2} s_{0} e^{ \pm i \hat{\chi} / 2}=s_{ \pm \chi} .
$$

In this equation, $\hat{\chi}$ is a diagonal matrix in channel space, with entries $\delta_{m, n} \chi_{m}$. Substitution into Eq. (55) gives

$$
\mathcal{Z}(\chi)=\operatorname{Det}\left[1+\left(\hat{s}_{-\chi}^{\dagger} \hat{s}_{\chi}-1\right) \hat{f}\right],
$$

in agreement with the existing literature. ${ }^{7}$

\section{TRACING OUT THE CHANNEL STRUCTURE}

A large class of experiments and devices in the field of quantum transport is based on two terminal setups. In such a setup, the channel space of the scatterer is naturally partitioned into a left and a right set, each connected to its own reservoir. We are generally interested in transport between left and right as opposed to internal dynamics on the left-or right-hand sides. The scattering matrices have the general structure

$$
\hat{s}_{ \pm}=X\left(\begin{array}{ll}
r & t^{\prime} \\
t & r^{\prime}
\end{array}\right) X^{-1}, \quad X=\left(\begin{array}{cc}
X_{L}^{ \pm} & \\
& X_{R}^{ \pm}
\end{array}\right) .
$$

Here, $r\left(r^{\prime}\right)$ describes left (right) to left (right) reflection, while $t\left(t^{\prime}\right)$ describes left (right) to right (left) transmission ( $t$ is not to be confused with time). These matrices have no time or Keldysh structure but still have a matrix structure in the space of left or right channel indices. The operators $X_{L}^{ \pm}(\tau)$ and $X_{R}^{ \pm}(\tau)$ have a diagonal Keldysh structure (denoted by the superscript \pm ) and a diagonal time structure (here indicated by $\tau$ to avoid confusion with the transmission matrix $t$ ). They do not have an internal channel structure and, as a result, the Keldysh action is insensitive to the internal dynamics on the left- or right-hand sides. Our shorthand for the Keldysh scattering matrix will be $\mathrm{Xs}_{s} \mathrm{X}^{-1}$, where we remember that $s$ has no Keldysh structure.

We now consider the square of the generating functional $\mathcal{Z}$ and employ the first expression we obtained for it [Eq. (49)], which retains the Keldysh structure in the determinant,

$$
\mathcal{Z}^{2}=\operatorname{Det}\left[\frac{1+G}{2}+X s X^{-1} \frac{1-G}{2}\right]^{2} \operatorname{Det} s^{\dagger} .
$$

Here, we exploited the fact that $\hat{s}_{-}$acts on half of Keldysh space together with the fact that $\hat{s}_{+}=\hat{s}_{-}$, i.e., $s$ has no Keldysh structure, to write $\exp 2 \operatorname{Tr} \ln \hat{s}_{-}=$Det $s$. We now shift $X$ to act on $G$ and define 


$$
\check{G}=X^{-1} G X, \quad P=\frac{1+\check{G}}{2}, \quad Q=\frac{1-\check{G}}{2} .
$$

The operators $P$ and $Q$ are complementary projection operators, i.e., $P^{2}=P, Q^{2}=Q, P Q=Q P=0$, and $P+Q=I$. Because of this, it holds that $\operatorname{Det}(P+s Q)=\operatorname{Det}(P+Q s)$. Thus, we find

$$
\mathcal{Z}^{2}=e^{2 \mathcal{A}}=\operatorname{Det}\left(P s^{\dagger}+s Q\right) .
$$

The left channels are all connected to a single reservoir, while the right channels are all connected to a different reservoir. This means that the reservoir Greens function has a channel space structure

$$
\check{G}=\left(\begin{array}{cc}
\check{G}_{L} & \\
& \check{G}_{R}
\end{array}\right),
$$

where $G_{L}$ and $G_{R}$ have no further channel space structure. At this point, it is worth explicitly stating the structure of operators carefully. In general, an operator carries Keldysh indices, indices corresponding to left and right, channel indices within the left or right sets of channels, and time indices. However $P, Q$, and $s$ are diagonal or even structureless, i.e., proportional to identity in some of these indices. Let us denote Keldysh indices with $k, k^{\prime} \in\{+,-\}$, left and right with $\alpha, \alpha^{\prime} \in\{L, R\}$, channel indices within the left or right sets with $c, c^{\prime} \in Z$, and time $t, t^{\prime} \in R$. Then $P$ has the explicit form

$$
P\left(k, k^{\prime} ; \alpha, \alpha^{\prime} ; c, c^{\prime} ; t, t^{\prime}\right)=P\left(k, k^{\prime} ; \alpha ; t, t^{\prime}\right) \delta_{\alpha, \alpha^{\prime}} \delta_{c, c^{\prime}} .
$$

The projection operator $Q$ has the same structure. The scattering matrix $s$ has the structure

$$
s\left(k, k^{\prime} ; \alpha, \alpha^{\prime} ; c, c^{\prime} ; t, t^{\prime}\right)=s\left(\alpha, \alpha^{\prime} ; c, c^{\prime}\right) \delta_{k, k^{\prime}} \delta\left(t-t^{\prime}\right) .
$$

We now use the formula

$$
\operatorname{Det}\left(\begin{array}{ll}
A & B \\
C & D
\end{array}\right)=\operatorname{Det}(A) \operatorname{Det}\left(D-C A^{-1} B\right)
$$

to eliminate the left-right structure from the determinant.

$$
\begin{aligned}
\mathcal{Z}^{2} & =\left(\begin{array}{cc}
P_{L} r^{\dagger}+Q_{L} r & P_{L} t^{\dagger}+Q_{R} t^{\prime} \\
P_{R} t^{\prime^{\dagger}}+Q_{L} t & P_{R} r^{\prime^{\dagger}}+Q_{R} r^{\prime}
\end{array}\right) \\
& =\operatorname{Det}\left(P_{L} r^{\dagger}+Q_{L} r\right) \operatorname{Det}\left[P_{R} r^{\prime \dagger}+Q_{R} r^{\prime}-\left(P_{R} t^{\prime \dagger}+Q_{L} t\right)\left(P_{L} r^{\dagger-1}+Q_{L} r^{-1}\right)\left(P_{L} t^{\dagger}+Q_{r} t^{\prime}\right)\right] \\
& =\underbrace{\operatorname{Det}\left(P_{L} r^{\dagger}+Q_{L} r\right)}_{a} \underbrace{\operatorname{Det}\left[P_{R}\left(r^{\prime \dagger}-P_{L} t^{\prime \dagger} r^{\dagger-1} t^{\dagger}\right)+\left(r^{\prime}-Q_{L} t r^{-1} t^{\prime}\right) Q_{R}-P_{R}\left(P_{L} t^{\prime \dagger} r^{\dagger-1} t^{\prime}+Q_{L} t^{\prime \dagger} r^{-1} t^{\prime}\right) Q_{R}\right]}_{b} .
\end{aligned}
$$

Here, it is important to recognize that the reflection and transmission matrices commute with the projection operators $P_{L, R}$ and $Q_{L, R}$. Furthermore, notice that, in term $b$, the projection operator $P_{R}$ always appears on the left of any product involving other projectors, while $Q_{R}$ always appears on the right. This means that in the basis where

$$
P_{R}=\left(\begin{array}{ll}
I & 0 \\
0 & 0
\end{array}\right), \quad Q_{R}=\left(\begin{array}{ll}
0 & 0 \\
0 & I
\end{array}\right),
$$

term $b$ is the determinant of an upper block-diagonal matrix. As such, it only depends on the diagonal blocks, so that the term $P_{R}(\ldots) Q_{R}$ may be omitted. Hence,

$$
b=\operatorname{Det}\left[P_{R}\left(r^{\prime \dagger}-P_{L} t^{\prime \dagger} r^{\dagger-1} t^{\dagger}\right)+\left(r^{\prime}-Q_{L} t r^{-1} t^{\prime}\right) Q_{R}\right] .
$$

Now we invoke the so-called polar decomposition of the scattering matrix, ${ }^{28}$

$$
\begin{gathered}
r=u \sqrt{1-T} u^{\prime}, \quad t^{\prime}=i u \sqrt{T} v, \\
t=i v^{\prime} \sqrt{T} u^{\prime}, \quad r^{\prime}=v^{\prime} \sqrt{1-T} v,
\end{gathered}
$$

where $u, u^{\prime}, v$, and $v^{\prime}$ are unitary matrices and $T$ is a diagonal matrix with the transmission probabilities $T_{n}$ on the di- agonal. We evaluate term $a$ in the basis where $P_{L}$ and $Q_{L}$ are diagonal to find

$$
a=\operatorname{Det}\left(\begin{array}{cc}
u^{\prime \dagger} \sqrt{1-T} u^{\dagger} & 0 \\
0 & u \sqrt{1-T} u^{\prime}
\end{array}\right)=\operatorname{Det}(I \sqrt{1-T}),
$$

where $I=P_{L}+Q_{L}=P_{R}+Q_{L}$ is the identity operator $I\left(k, k^{\prime} ; c, c^{\prime} ; t, t^{\prime}\right)=\delta_{k, k^{\prime}} \delta_{c, c^{\prime}} \delta\left(t-t^{\prime}\right)$ in Keldysh, channel, and time indices. For term $b$, we find

$$
\begin{aligned}
b= & \operatorname{Det}\left[P_{R}\left(\sqrt{1-T}+P_{L} \frac{T}{\sqrt{1-T}}\right)\right. \\
& \left.+\left(\sqrt{1-T}+Q_{L} \frac{T}{\sqrt{1-T}}\right) Q_{R}\right] .
\end{aligned}
$$

Combining the expressions for $a$ and $b$, we find

$$
\mathcal{Z}^{2}=e^{2 \mathcal{A}}=\operatorname{Det}\left[1-T\left(P_{R} Q_{L}+P_{L} Q_{R}\right)\right] .
$$

Using the fact that $P_{L(R)}=\left(1+\check{G}_{L(R)}\right) / 2$ and $Q_{L(R)}=(1$ $\left.-\check{G}_{L(R)}\right) / 2$ and taking the logarithm, we finally obtain the remarkable result 


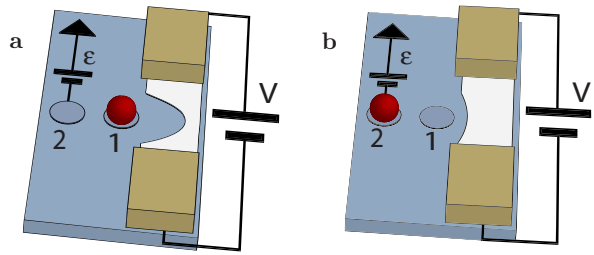

FIG. 2. (Color online) A schematic picture of the system considered. It consists of a charge qubit coupled to a QPC. The shape of the QPC constriction, and hence its scattering matrix, depends on the state of the qubit. A gate voltage controls the qubit level splitting $\varepsilon$. There is a small tunneling rate $\gamma$ between qubit states.

$$
\mathcal{A}=\frac{1}{2} \sum_{n} \operatorname{Tr} \ln \left[1+\frac{T_{n}}{4}\left(\left\{\check{G}_{L}, \check{G}_{R}\right\}-2\right)\right] .
$$

This formula was used in Ref. 14 to study the effects on transport of electromagnetic interactions among electrons. In Ref. 16, the same formula was employed to study the output of a two-level measuring device coupled to the radiation emitted by a quantum point contact (QPC).

\section{FERMI-EDGE SINGULARITY}

In this section, we show how our formulas apply to a phenomenon known as the Fermi-edge singularity. The system under consideration is one of the most elementary examples of an interacting electron system. The initial analysis ${ }^{8,9}$ relied on diagrammatic techniques rather than on the scattering approach or the Keldysh technique, and was confined to equilibrium situations. Several decades later, the problem was revisited in the context of the scattering approach. ${ }^{12,13}$ An intuitive derivation of a determinant formula was given. Here, we apply our approach to confirm the validity of this previous work. We find exact agreement. This highlights the fact that the determinant formulation of the FES problem is also valid for multichannel devices out of equilibrium, an issue not explicitly addressed in the existing literature.

The original problem ${ }^{8,9}$ was formulated for conduction electrons with a small effective mass and valence electrons with a large effective mass, bombarded by $\mathrm{x}$ rays. The $\mathrm{x}$ rays knock one electron out of the valence band, leaving behind an essentially stationary hole. Until the hole is refilled, it interacts through the Coulomb interaction with the conduction electrons. The $\mathrm{x}$-ray absorption rate is studied. Abanin and Levitov reformulated the problem in the context of quantum transport, where an electron tunnels into or out of a small quantum dot that is side-coupled to a set of transport channels.

We prefer to consider a slightly simpler setup that exhibits the same physics. The setup is illustrated in Fig. 2. A QPC interacts with a charge qubit. The shape of the QPC constriction depends on the state of the qubit. The Hamiltonian for the system is

$$
\mathcal{H}=\mathcal{H}_{1}|1\rangle\left\langle 1\left|+\left(\mathcal{H}_{2}+\varepsilon\right)\right| 2\right\rangle\langle 2|+\gamma(|1\rangle\langle 2|+| 2\rangle\langle 1|) .
$$

The operators $\mathcal{H}_{1}\left(\mathcal{H}_{2}\right)$ describe the QPC electrons when the qubit is in state $|1\rangle(|2\rangle)$. They differ by a potential energy term, describing the pinching off of the QPC constriction depending on the state of the qubit. We may take both Hamiltonians to be of the form [Eq. (5)] that we wrote down for a general scatterer. The energy $\varepsilon$ is the qubit level splitting, an experimentally tunable parameter. The QPC may or may not be driven by a voltage bias $V$.

QPC electrons do not directly interact with each other, but rather with the qubit. This interaction is the only qubit relaxation mechanism included in our model. We work in the limit $\gamma \rightarrow 0$, where the inelastic transition rates $\Gamma_{12,21}$ between qubit states are small compared to the energies $\mathrm{eV}$ and $\varepsilon$. In this case, the qubit switching events can be regarded as independent and incoherent.

Now consider the qubit transition rate $\Gamma_{21}$ from state $|1\rangle$ to $|2\rangle$ as a function of the qubit level splitting $\varepsilon$. To lowest order in the tunneling amplitude $\gamma$, it is given by

$$
\begin{gathered}
\Gamma_{21}=2 \gamma^{2} \operatorname{Re} \int_{-\infty}^{0} d \tau e^{i \varepsilon \tau} \lim _{t_{0} \rightarrow-\infty} \exp \mathcal{A}(\tau), \\
\exp \mathcal{A}(\tau)=\operatorname{Tr}\left[e^{i \hat{H}_{2} \tau} e^{-i \hat{H}_{1}\left(\tau-t_{0}\right)} \rho_{0} e^{-i \hat{H}_{1} t_{0}}\right] .
\end{gathered}
$$

This is the usual Fermi golden rule. The time $\tau$ over which we integrate can be interpreted as the time when the qubit switches from $|1\rangle$ to $|2\rangle$. The trace is over QPC states, and $\rho_{0}$ is the initial QPC density matrix. We see that the expression for $\Gamma_{21}$ contains an instance of the Keldysh action $\mathcal{A}$ that we have calculated. The correspondence requires us to set

$$
\begin{gathered}
\mathcal{H}^{+}(t)=\mathcal{H}_{1}+\left(\mathcal{H}_{2}-\mathcal{H}_{1}\right) \theta(t-\tau) \theta(-t), \\
\mathcal{H}^{-}(t)=\mathcal{H}_{1} .
\end{gathered}
$$

In order to conform to the conventions of the existing literature, we write $\mathcal{Z}$ in the form where the Keldysh structure has been removed [Eq. (4)]:

$$
\mathcal{A}(\tau)=\operatorname{Tr} \ln \left[\hat{s}_{-}(1-\hat{f})+\hat{s}_{+}(\tau) \hat{f}\right]-\operatorname{Tr} \ln \hat{s}_{-} .
$$

In this formula, $\hat{s}_{-}$is the scattering matrix corresponding to $\mathcal{H}^{-}=\mathcal{H}_{1}$ when the qubit is in state $|1\rangle$. It is proportional to identity in time indices. The scattering matrix $\hat{s}_{+}(\tau)$ corresponds to $\mathcal{H}^{+}$. It is still diagonal in time indices, but the diagonal elements $\hat{s}_{+}(\tau)_{t}$ are time dependent. If we take the time it takes an electron to traverse the conductor to be much shorter than other time scales, such as the attempt rate of charge transfers, then

$$
\hat{s}_{+}(\tau)_{t}=\hat{s}_{1}+\left(\hat{s}_{2}-\hat{s}_{1}\right) \theta(t-\tau) \theta(-t),
$$

where $\hat{s}_{2}$ is the scattering matrix associated with $\mathcal{H}_{2}$ when the qubit is in state $|2\rangle$. This expression first appeared in Ref. 12. In the language of the original diagrammatic treatment of the FES problem, ${ }^{8,9}$ it represents the total closed loop contribution.

We may also write this closed loop contribution as

$$
e^{\mathcal{A}(\tau)}=\operatorname{Det}\left[1+\left(\hat{s}_{1}^{\dagger} \hat{s}_{2}-1\right) \hat{\Pi}(\tau) \hat{f}\right],
$$

where $\hat{\Pi}$ is a diagonal operator in time domain with a kernel that is a double step function, 


$$
\Pi(\tau)_{t, t^{\prime}}=\theta(-t) \theta(t-\tau)
$$

and the scattering matrices $\hat{s}_{1}$ and $\hat{s}_{2}$ no longer have any time structure. We may work in the channel space basis where $\hat{s}_{1}^{\dagger} \hat{s_{2}}$ is diagonal. Its eigenvalues are $e^{i \lambda_{k}}$. Suppose we are in zero-temperature equilibrium, then the filling factor $f$ is the same in every channel. In the Fourier transformed energy basis, $f$ is simply a step function:

$$
f_{\varepsilon, \varepsilon^{\prime}}=\delta\left(\varepsilon-\varepsilon^{\prime}\right) \theta(-\varepsilon) .
$$

Thus, one finds

$$
e^{\mathcal{A}}=\prod_{k} \operatorname{Det}\left[1+\left(e^{i \lambda_{k}}-1\right) \hat{\Pi}(\tau) \hat{f}\right]
$$

This determinant contains no channel structure any more. Operators only have one set of indices (time or, after Fourier transform, energy). $\hat{\Pi}$ is a projection operator, diagonal in time domain, while $\hat{f}$ is a projection operator in energy domain. Such a determinant is known as a Fredholm determinant.

The resulting transition rate is ${ }^{8,9,12}$

$$
\Gamma_{21}(\varepsilon)=\theta(-\epsilon) \frac{1}{|\epsilon|}\left(\frac{|\varepsilon|}{E_{\mathrm{co}}}\right)^{\alpha},
$$

where $E_{\mathrm{co}}$ is a cutoff energy of the order of the Fermi energy measured from the bottom of the conduction band. The exponent $\alpha$ is known as the orthogonality exponent. It may be calculated by analytically evaluating the Fredholm determinant with the Wiener-Hopf method. It is given in terms of the scattering matrices as ${ }^{11,12}$

$$
\alpha=\frac{1}{4 \pi^{2}}\left|\operatorname{Tr} \ln ^{2}\left(s_{1}^{\dagger} s_{2}\right)\right|,
$$

with the trace being over channel indices. Inspired by the work of Abanin and Levitov, ${ }^{12,13}$ we considered the case where the QPC is driven by a voltage bias. The results of our study may be found in Ref. 17.

\section{CONCLUSION}

In this paper, we have derived several expressions for the Keldysh action $\mathcal{A}$ for a general multiterminal, timedependent scatterer. This object is defined as the (logarithm of the) trace of the density matrix of the scatterer after an evolution forward and backward in time with different Hamiltonians:

$$
\begin{aligned}
e^{\mathcal{A}}= & \operatorname{Tr}\left[\mathcal{T}^{+} \exp \left\{-i \int_{t_{0}}^{t_{1}} d t \mathcal{H}^{+}(t)\right\}\right. \\
& \left.\times \rho_{0} \mathcal{T}^{-} \exp \left\{i \int_{t_{0}}^{t_{1}} d t \mathcal{H}^{-}(t)\right\}\right] .
\end{aligned}
$$

Our main result is a compact formula for the action in terms of reservoir Green's functions and the scattering matrix of the scatterer [Eq. (1)]. We have shown how to explicitly perform the trace over Keldysh indices when reservoirs are characterized by filling factors. Thus, we obtained a formula [Eq. (2)] generalizing the Levitov counting statistics formula. We have also explicitly performed the trace over channel indices for a two terminal scatterer [Eq. (4)]. In this case, we demonstrated that the Keldysh action only depends on the scattering matrix through the eigenvalues of the transmission matrix. To illustrate the utility of the Keldysh action, and confirm the correctness of our results, we considered full counting statistics and the Fermi-edge singularity. We found that our results agree with the existing literature.
${ }^{1}$ R. Landauer, IBM J. Res. Dev. 1, 223 (1957).

${ }^{2}$ M. Büttiker, Y. Imry, R. Landauer, and S. Pinhas, Phys. Rev. B 31, 6207 (1985).

${ }^{3}$ M. Büttiker, Phys. Rev. Lett. 65, 2901 (1990).

${ }^{4}$ M. Büttiker, Phys. Rev. B 46, 12485 (1992).

${ }^{5}$ G. B. Lesovik, JETP Lett. 49, 592 (1989).

${ }^{6}$ L. S. Levitov and G. B. Lesovik, JETP Lett. 58, 230 (1993).

${ }^{7}$ L. S. Levitov, H.-W. Lee, and G. B. Lesovik, J. Math. Phys. 37, 10 (1996).

${ }^{8}$ G. D. Mahan, Phys. Rev. 163, 612 (1967).

${ }^{9}$ P. Nozières and C. T. De Dominicic, Phys. Rev. 178, 1097 (1969)

${ }^{10}$ K. A. Matveev and A. I. Larkin, Phys. Rev. B 46, 15337 (1992).

${ }^{11}$ K. Yamada and K. Yosida, Prog. Theor. Phys. 68, 1504 (1982).

${ }^{12}$ D. A. Abanin and L. S. Levitov, Phys. Rev. Lett. 93, 126802 (2004).

${ }^{13}$ D. A. Abanin and L. S. Levitov, Phys. Rev. Lett. 94, 186803 (2005).

${ }^{14}$ M. Kindermann and Yu. V. Nazarov, Phys. Rev. Lett. 91, 136802 (2003)

${ }^{15}$ M. Kindermann, Yu. V. Nazarov, and C. W. J. Beenakker, Phys. Rev. B 69, 035336 (2004).
${ }^{16}$ J. Tobiska, J. Danon, I. Snyman, and Yu. V. Nazarov, Phys. Rev. Lett. 96, 096801 (2006).

${ }^{17}$ I. Snyman and Yu. V. Nazarov, Phys. Rev. Lett. 99, 096802 (2007).

${ }^{18}$ C. W. J. Beenakker, in Transport Phenomena in Mesoscopic Systems, edited by H. Fukuyama and T. Ando (Springer, New York, 1992).

${ }^{19}$ R. P. Feynman and F. L. Vernon, Ann. Phys. (N.Y.) 24, 118 (1963).

${ }^{20}$ L. V. Keldysh, Zh. Eksp. Teor. Fiz. 47, 1515 (1964).

${ }^{21}$ Yu. V. Nazarov, Superlattices Microstruct. 25, 1221 (1999).

${ }^{22}$ Yu. V. Nazarov, Phys. Rev. Lett. 73, 1420 (1994).

${ }^{23}$ G. Schön and A. D. Zaikin, Phys. Rep. 198, 237 (1990).

${ }^{24}$ Yu. V. Nazarov, Phys. Rev. Lett. 82, 1245 (1999).

${ }^{25}$ Yuli V. Nazarov, in Handbook of Theoretical and Computational Nanotechnology, edited by M. Rieth and W. Schommers (American Scientific, Stevenson Ranch, CA, 2006).

${ }^{26}$ K. B. Efetov, Supersymmetry in Disorder and Chaos (Cambridge University Press, Cambridge, 1999); M. V. Feigel'man, A. I. Larkin, and M. A. Skvortsov, Phys. Rev. B 61, 12361 (2000).

${ }^{27}$ J. Rammer and H. Smith, Rev. Mod. Phys. 58, 323 (1986).

${ }^{28}$ H. U. Baranger and P. A. Mello, Phys. Rev. Lett. 73, 142 (1994). 